\title{
Establishing Minimal Clinically Important Differences for the Quality of Life Instrument in Patients with Esophageal Cancer QLICP-ES (V2.0) Based on Anchor-Based and Distribution-Based Methods
}

Ting Wu

Guangdong Medical University

Dandan Ren

Guangdong Medical University

Yanbo Qi

the Center for Disease Control and Prevention of Yunan Province

Yujing Fang

Sun Yat-sen University Cancer Center

Jiudi Zhong

Sun Yat-sen University Cancer Center

Gaofeng Li

Yunan Tumor Hospital

chonghua wan ( $\square$ wanchh@hotmail.com)

Guangdong Medical University

Research article

Keywords: esophageal cancer, quality of life, MCID, anchor-based methods, distribution-based methods

Posted Date: August 20th, 2020

DOl: https://doi.org/10.21203/rs.3.rs-57319/v1

License: (c) (i) This work is licensed under a Creative Commons Attribution 4.0 International License. Read Full License 


\section{Abstract}

Background: The minimal clinically important difference (MCID) is an important phrase with big appeal in a field struggling to interpret quality of life (QOL) and other patient-reported outcomes (PRO), is also a bridge between statistics and clinical medicine. This paper is aimed to determine the MCID of esophageal cancer scale among Quality of Life Instruments system for Cancer Patients, QLICP-ES (V2.0).

Methods: According to the scoring rule of QLICP-ES (V2.0), the scores of each domain and the overall of the scale were calculated. The MCID values of this scale were established by anchor-based and distributionbased methods. Two criteria A (improves one level after treatments) and B (at least improves one levels after treatments) were defined treatments effects in anchor-based methods, while methods of ES, SEM and $\mathrm{RCl}$ were used in distribution-based methods.

Results: Using the anchor-based method, according to standard A, the MCID values of physical domain, psychological domain, social domain, common symptom and side-effects domain, the specific domain and the overall were $15.1,4.4,3.1,6.7,8.5$ and 6.0 respectively. According to standard $B$, the MCID values of above domains and the overall were $19.3,4.2,4.8,7.7,9.5$ and 7.5 respectively. Under the distribution-based methods, the MCID values above calculated by each method (ES, SEM and RCI) are in different ranges from 1.1 to 13.3 .

Conclusion: All methods have its own advantages and disadvantages to develop the MCID values, so it is necessary to develop the MCID values of QLICP-ES (V2.0) comprehensively with a variety of methods considering the actual situation.

\section{Background}

Esophageal cancer (ES) is the sixth most common cancer in the world and the fourth most common cause of cancer deaths in China. Nearly half a million new ES cases are diagnosed each year [1,2]. In China, the incidence of esophageal cancer ranks the sixth among malignant tumors, and the incidence of male cancer is higher than female cancer, and the incidence of rural cancer is higher than urban cancer [3]. The clinical symptoms of early esophageal cancer are not obvious, and dysphagia is often the main symptom, which may be combined with gastrointestinal and other system-related symptoms [4], resulting in somatic or functional damage, which seriously affects the physical and mental health of patients and reduces their quality of life (QOL) and other patient-reported outcomes (PRO). With the medical model entering the biopsycho-social medical model (modern medical model), people pay more attention to the improvement of QOL while paying more attention to the prolonging of life.

Therefore, the assessments of QOL have been applied as significant outcome indicators for patients with ES [5.6], given the time course of the disease, the difficulty in curing and the burden of treatments. Consequently, several specific instruments for patients with ES have been developed and are used in cancer clinical researches, including European Organization for Research and Treatment of Cancer (EORTC) Quality of Life Group questionnaire, the QLQ-OES23 [7.8], the Functional Assessment of Cancer TherapyEsophageal cancer questionnaire (FACT-E) [9]. In China, the QOL team led by professor Chonghua Wan 
developed a scale system called QLICPs (Quality of Life Instruments for Cancer Patients), in which the QLICP-ES (the first version and the second version) is an instrument for quality of life measurement of esophageal cancer[10,11]. The QLICPs is a Chinese QOL instruments system developed by module approach with a general module (QLICP-GM) being used with all types of cancer, and some specific modules for different cancers $[12,13]$.

The QLICP-ES (V2.0) includes a generic module QLICP-GM (V2.0) and an esophageal cancer specific module, with the QLICP-GM (V2.0) including physical function (8 items), psychological (9 items), social functions ( 8 items), common symptoms and side effects ( 7 items), a total of 10 side and 32 items in 4 domains. The esophageal cancer specific module includes four domains and 16 items. The whole scale consists of 14 facets and 48 items in 5 domains (dimensions), which can be used for patients with various types of esophageal cancer, and can be used for the measurement of patients' quality of life in the period of onset, treatment and rehabilitation.

For reasonable explanation of the actual clinical significance of questionnaire survey and the scale score, the minimal clinically important differences (MCID) as an important change assessment tools or scale responsiveness of appropriate benchmarks have been proposed for the first time in 1987. Canadian scholar Jaeschke and others defined MCID as the minimum variation of questionnaire dimension score acceptable to patients without considering side effects and costs $[14,15]$.

The MCID is an important phrase with big appeal in a field struggling to interpret QOL and other patientreported outcomes (PRO), is also a bridge between statistics and clinical medicine. How to develop reasonable and reliable MCID values has become a hot topic for scholars. There are mainly two traditional methods, including anchor-based method and distribution-based method. At present, although QLICP-ES (V2.0) has been developed, MCID has not been developed. For this reason, in this paper, the MCID of QLICPES (V2.0) was developed by using the commonly used anchor-based method and distribution-based method, and the advantages and disadvantages of the two methods were compared to lay a foundation for clinical application.

\section{Methods}

\section{Data sources}

In this study, 232 inpatients with esophageal cancer diagnosed by pathological examination and diagnosed by thoracic surgery in a provincial cancer hospital were selected as the research objects. The inclusion and exclusion criteria are as follows:

Inclusion criteria: (1)Patients diagnosed with esophageal cancer by X-ray and fiberoptic esophagoscopy or gastroscopy biopsy; (2) Inpatients receiving treatments for esophageal cancer; (3) Understanding of the questionnaire due to the fact that the patient himself have a primary school education or above; (4)Patients who voluntarily participated in the test on this quality of life scale[16]. 
Exclusion criteria: (1) Patients who are illiterate or lack the ability to read and write;(2) Patients who are unable to express their true feelings clearly due to vague consciousness during hospitalization;(3) Patients who are unable to participate the test due to suffering from other serious diseases.

The QLICP-ES (V2.0) scale and EORTC QLQ-C30 were given to 232 patients with esophageal cancer before and after treatments, and was filled in once before and after treatments.

\section{Anchor-based methods}

The anchor-based method is to clarify the meaning of the scale score change by examining the relationship between the scale and another independent measurement tool score or other indicators. There are crosssectional anchors and longitudinal anchors. In this study, longitudinal anchors were selected to compare the curative effect before and after treatments. First, the Raw Score (RS) of domains were computed according to the number of questions and the answers of patients in each domain. Then, linear transformation is carried out with range method to convert the original score into a standardized score within 0-100.The score calculation method of each domain is as follows[17]:

$$
S S=(R S-M \text { in }) \times 100 / R \quad R=\text { Max }- \text { Min }
$$

Second, the Q29 item in the EORTC QLQ-C30 scale, "how would you evaluate your overall health in the past week", was selected as an anchor after considering the correlation coefficient between Q29 and QLICP-ES (V2.0) . Then, patients with a difference of one grade (standard A) and at least one grade (standard B) in Q29 before and after treatments were selected, and the score differences in various domains before and after treatments were calculated respectively, and the mean value of the difference was denoted as MCID.

\section{Distribution-based methods}

The distribution method is to determine the MCID from a statistical point of view by using the distribution(variation) of the sample data of the evaluation tool. The commonly used indexes to calculate variation include Effect-Size (ES), Standard Error of the Measurement (SEM), and Reliable Change Index $(\mathrm{RCl})$.

Effect-Size (ES) is obtained by dividing the difference in mean scores from baseline ${ }_{0}$ to post-intervention 1 by the standard-deviation of the baseline score ( $\left.\mathrm{SD}_{\text {baseline }}\right)$ [18], the calculation formula and the corresponding MCID are as follows:

$$
E S=\frac{\bar{x}_{1}-\bar{x}_{0}}{\sqrt{\sum\left(x_{0}-\bar{x}_{0}\right)^{2} / n-1}} \quad M C I D=E S \times S D_{\text {baseline }}
$$

ES is often used to compare two or more groups to measure the size of the difference between groups. In health-related quality of life assessments, ES is currently the most recognized parameter in determining the 
importance of group or individual changes. Cohen [19] empirically defined an effect size of 0.2 as small,0.5 as moderate, and 0.8 as large.

Standard Error of the Measurement (SEM), defined as the baseline SD multiplied by the square root of one minus sample test-retest reliability coefficient, were also calculated for comparison purpose [20]. The reliability is usually estimated using a test-retest reliability estimate, but some authors also use an internal consistency estimate, for example Cronbach's alpha [21]. The calculation formula and the corresponding MCID are as follows:

$$
S E M=\sqrt{1-r} \times \sqrt{\sum\left(x_{0}-\bar{x}_{0}\right)^{2} / n-1} \quad M C I D=X \times S D_{\text {baseline }} \times \sqrt{1-r}
$$

SEM is assumed to be fairly sample-independent [22], which is its best advantage: a growing standard deviation is balanced by a higher reliability. Some authors like Wyrwich et al. consider one SEM as an approximation of the MCID $[23,24]$. $X$ can be assigned to 1 (small effect), 1.96 (medium effect), 2.77(large effect).

Reliable Change Index $(\mathrm{RCl})$ is the change value of the questionnaire score divided by the square root of SEM. If $\mathrm{RCl}$ is greater than 1.96 , then the change value has a $95 \%$ chance of being a meaningful change [25]. The calculation formula and the corresponding MCID are as follows:

$$
R C I=\frac{\bar{x}_{1}-\bar{x}_{0}}{\sqrt{2(S E M)^{2}}} \quad M C I D=X \times S D_{\text {baseline }} \times \sqrt{2(1-\mathrm{r})}
$$

\section{Results}

\section{The demographic characteristics of the sample}

Table 1 showed the general demographic characteristics of the sample. A total of 232 patients with esophageal cancer were investigated in this study. There were 204 males and 28 females. The age distribution was 35-82 years old, with the mean of 59.3(SD8.9). Male elderly patients are more common. The occupations were mostly workers and farmers, with 20 workers (20.0\%) and 35 farmers (35.0\%) respectively. The educational level varies, among which Middle school or High school has the highest proportion(55.6\%). 
Table 1

Socio-demographic and clinical characteristics of the Sample $(n=232)$

\begin{tabular}{|c|c|c|c|c|c|}
\hline Characteristics & $\mathbf{N}$ & $\%$ & Characteristics & $\mathbf{N}$ & $\%$ \\
\hline Gender & & & Marital status & & \\
\hline Male & 204 & 87.9 & Married & 226 & 97.4 \\
\hline Female & 28 & 12.1 & Others & 6 & 2.6 \\
\hline Age & & & Ethnicity & & \\
\hline $30-39$ & 3 & 1.3 & Han & 203 & 87.5 \\
\hline $40-49$ & 32 & 13.8 & Others & 29 & 12.5 \\
\hline $50-59$ & 67 & 28.9 & Education & & \\
\hline$\geq 60$ & 130 & 56.0 & Primary school & 81 & 34.9 \\
\hline \multirow[t]{2}{*}{ Average $^{\star}$} & \multicolumn{2}{|c|}{$59.3(8.9)$} & Middle school or High school & 129 & 55.6 \\
\hline & & & College/University & 22 & 9.5 \\
\hline Perceived Income & & & Medical insurance & & \\
\hline Poor & 52 & 22.4 & Self-paid/Private insurance & 4 & 1.7 \\
\hline Fair & 139 & 59.9 & Medical insurance & 228 & 98.3 \\
\hline High & 41 & 17.7 & & & \\
\hline Occupation & & & Treatments & & \\
\hline Worker & 62 & 26.7 & Surgery & 134 & 57.8 \\
\hline Farmer & 74 & 31.9 & chemotherapy & 51 & 22.0 \\
\hline Others & 96 & 41.4 & Others & 31 & 13.4 \\
\hline
\end{tabular}

\section{The MCID values using Anchor-based methods}

Table 2 showed different MCID values obtained with anchor-based methods. With the Q29 item in the EORTC QLQ-C30 scale as anchor, "how would you evaluate your overall health in the past week", the correlation coefficient between Q29 and the score of QLICP-ES (V2.0), was calculated $r=0.75$. The correlation coefficients between Q29 and other domains (physical domain, psychological domain, social domain, common symptom and side-effects domain and the specific domain) were $0.69,0.17,0.32,0.57$ and 0.68 respectively. Then according to standard A, 102 patients with exactly one grade difference in Q29 before and after treatments were selected, and the difference of scores in all domains and total scale of QLICP-ES (V2.0) before and after treatments was calculated. In the same method, 165 patients with at least one grade 
difference in Q29 before and after treatments were selected according to standard B, and the difference of scores in all domains and total scale of QLICP-ES (V2.0) before and after treatments was calculated. The mean standard deviations of the difference scores in each domain and the total scale under the two standards were calculated, and the mean values of the difference scores were recorded as MCID.

Table 2

The MCID value of QLICP-ES (V2.0) determined by anchor-based method $\left(n_{A}=102, n_{B}=165\right)$

\begin{tabular}{|llllll|}
\hline Domain & Items & $\begin{array}{l}\text { Standard } \\
\text { A }\end{array}$ & $\begin{array}{l}\text { Standard } \\
\text { B }\end{array}$ & $\begin{array}{l}\text { Standard } \\
\text { A MCID }\end{array}$ & $\begin{array}{l}\text { Standard } \\
\text { B MCID }\end{array}$ \\
\hline Physical domain (PHD) & 8 & $15.1 \pm 14.8$ & $19.3 \pm 16.1$ & 15.1 & 19.3 \\
\hline Psychological domain (PSD) & 9 & $-4.4 \pm 11.8$ & $-4.2 \pm 12.4$ & 4.4 & 4.2 \\
\hline Social domain (SOD) & 8 & $3.1 \pm 10.2$ & $4.8 \pm 11.2$ & 3.1 & 4.8 \\
\hline $\begin{array}{l}\text { Common symptoms and side effect } \\
\text { domain (SSD) }\end{array}$ & 7 & $6.7 \pm 10.7$ & $7.7 \pm 11.3$ & 6.7 & 7.7 \\
\hline Core/general module (CGM) & 32 & $4.8 \pm 6.5$ & $6.5 \pm 7.3$ & 4.8 & 6.5 \\
\hline Specific domain (SPD) & 16 & $8.5 \pm 8.3$ & $9.5 \pm 9.2$ & 8.5 & 9.5 \\
\hline Total (TOT) & 48 & $6.0 \pm 6.0$ & $7.5 \pm 6.8$ & 6.0 & 7.5 \\
\hline
\end{tabular}

\section{The MCID values using Distribution-based methods}

The distribution method estimates MCID values based on the observed distribution of score changes, and the distribution variation can be measured using several statistical effects. Three variation indexes, EffectSize (ES), Standard Error of the Measurement (SEM) and Reliable Change Index (RCI), were used to calculate the MCID values of esophageal cancer in this study.

Table 3 showed the MCID values calculated using ES method under different effects $(X$ of $0.2,0.5$ and 0.8 were used to define small, medium and large effects) in detail. 
The MCID value of QLICP-ES (V2.0) was determined by ES\n=232》

\begin{tabular}{|lllll|}
\hline Domain & SD $_{\text {baseline }}$ & $\mathbf{0 . 2 E S}$ & $\mathbf{0 . 5 E S}$ & $\mathbf{0 . 8 E S}$ \\
\hline Physical domain (PHD) & 15.9 & 3.2 & 8.0 & 12.7 \\
\hline Psychological domain (PSD) & 13.7 & 2.7 & 6.8 & 10.9 \\
\hline Social domain (SOD) & 11.8 & 2.4 & 5.9 & 9.5 \\
\hline Common symptoms and side effect domain (SSD) & 14.3 & 2.9 & 7.1 & 11.4 \\
\hline Core/general module (CGM) & 9.2 & 1.8 & 4.6 & 7.4 \\
\hline Specific domain (SPD) & 14.3 & 2.9 & 7.1 & 11.4 \\
\hline Total (TOT) & 9.7 & 2.0 & 4.9 & 7.8 \\
\hline
\end{tabular}

Some scholars believe that the Standard Error of the Measurement (SEM) is not completely dependent on the sample information and maintains a certain stability between different studies, which is better than the Effect-Size (ES). Wyrwich[24] et al. considered that 1SEM could be regarded as the minimum change value of clinical significance, and some scholars conservatively used 1.96SEM as the minimum change value of clinical significance $[26,27]$. Table 4 and Table 5 showed the MCID values calculated using SEM and RCI methods in detail.

Table 4

The MCID value of QLICP-ES (V2.0) determined by SEM ( $n=232)$

\begin{tabular}{|lllll|}
\hline Domain & $\mathbf{r}$ & $\mathbf{S D}_{\text {baseline }}$ & SEM & 1.96 SEM \\
\hline Physical domain (PHD) & 0.98 & 15.9 & 2.1 & 4.2 \\
\hline Psychological domain (PSD) & 0.96 & 13.7 & 2.9 & 5.7 \\
\hline \multicolumn{1}{|c|}{ Social domain (SOD) } & 0.92 & 11.8 & 3.4 & 6.7 \\
\hline Common symptoms and side effect domain (SSD) & 0.99 & 14.3 & 1.6 & 3.1 \\
\hline Core/general module (CGM) & 0.99 & 9.2 & 1.1 & 2.2 \\
\hline Specific domain (SPD) & 0.96 & 14.3 & 2.8 & 5.5 \\
\hline Total (TOT) & 0.99 & 9.7 & 1.1 & 2.2 \\
\hline
\end{tabular}


The MCID value of QLICP-ES (V2.0) determined by RCI $(\mathrm{n}=232)$

\begin{tabular}{|llllll|}
\hline Domain & $\mathbf{r}$ & $\mathbf{S D}_{\text {baseline }}$ & $\mathbf{R C l}$ & $\mathbf{1 . 9 6 R C I}$ & $\mathbf{2 . 7 7 R C I}$ \\
\hline Physical domain (PHD) & 0.98 & 15.9 & 3.0 & 5.9 & 8.4 \\
\hline Psychological domain (PSD) & 0.96 & 13.7 & 4.1 & 8.0 & 11.4 \\
\hline Social domain (SOD) & 0.92 & 11.8 & 4.8 & 9.4 & 13.3 \\
\hline Common symptoms and side effect domain (SSD) & 0.99 & 14.3 & 2.2 & 4.3 & 6.1 \\
& & & & & \\
\hline Core/general module (CGM) & 0.99 & 9.2 & 1.6 & 3.1 & 4.4 \\
\hline Specific domain (SPD) & 0.96 & 14.3 & 3.9 & 7.7 & 10.9 \\
\hline Total (TOT) & 0.99 & 9.7 & 1.6 & 3.1 & 4.4 \\
\hline
\end{tabular}

\section{Discussion}

The main function of MCID is to assist researchers and clinical staff to explain the significance of changes or differences in the score of evaluation tools. MCID values can be used to determine whether changes in the score of the scale brought by clinical interventions have "clinical significance"[28]. For clinicians and researchers, the key for using measurement instruments is the effectiveness and stability of the MCID score. Lower MCID values may lead to overestimation of the positive effects of treatment, while higher MCID values may incorrectly classify patients as ineffective when the treatment is actually beneficial. There are various methods to calculate MCID for esophageal cancer. Using the anchor-based method, according to standard A, the MCID values of physical domain, psychological domain, social domain, common symptom and side-effects domain, the specific domain and the overall were 15.1, 4.4, 3.1, 6.7, 8.5 and 6.0 respectively. According to standard B, the MCID values of above domains and the overall were 19.3, 4.2, 4.8, 7.7, 9.5 and 7.5 respectively in this study.

The correlation coefficient $r$ between the selected anchor Q29 and the score of the total scale was 0.72 , but the correlation coefficient between the anchor Q29 and some domains, such as psychological domain and social domain, was 0.17 and 0.32 , which were not too high. Therefore, the distribution-based methods were further adopted and the MCID values under ES, SEM and RCI were calculated respectively. Studies have shown that when calculating MCID values by ES method, 0.5 medium effect was the most appropriate, while 0.2 and 0.8 were easy to be too large or too small in calculation [29]. And the results are closer to the MCID values calculated by the anchor-based method. SEM is not completely dependent on the sample information and is slightly affected by the sample size. Therefore, scholars recommend 1SEM or 1.96 SEM as MCID values.

Anchor-based methods and distribution-based method have their own advantages and disadvantages, so this study used a variety of methods to calculate the MCID values. The advantage of the anchor-based methods is that the change in the outcome measure score is associated with a meaningful external anchor 
that reflects the patient's point of view and provides a professional explanation for the minimum change in clinical significance determined. However, the disadvantage is that the measurement error is not taken into account, different criteria will produce different changes in the minimum clinical significance, and it is often difficult to find a suitable anchor. Moreover, in this paper, the sample size is not too large and only subjective anchors are used, subsequent studies will expand the sample size and use objective anchors for further studies. The distribution-based methods take measurement error into account to determine the MCID values, which is relatively easy to implement, but the results obtained from different samples may be different, so the professional explanation cannot be given for the determined minimum measurable change value, and there is a lack of recognized judgment criteria for the obtained minimum measurable change values. In developing MCID, the anchor-based method is generally preferred. When there are no good criteria or a small sample size, the distribution-based methods will be considered comprehensively.

MCID is not a fixed value, and different determination methods can produce different MCID values. Not all methods produce similar MCID values, and not all MCID values are generic. The interpretation and application of MCID values should be carefully considered in combination with the actual clinical situation. In this paper, a lot of different MCID values were presented so that it can be easy and convenient to select by users.

Although MCID as a minimum threshold plays an important role in determining the scale score changes, its stability and variability may be affected by many factors, such as defects in the measurement method, demographic characteristics, patient comprehension and study cycles and so on [30].

\section{Conclusion}

In conclusion, appropriate methods should be adopted to evaluate the MCID of each scale. When MCID is applied to evaluate the clinical efficacy of esophageal cancer, it is necessary to carefully review and fully understand the concepts and influencing factors of each method, so as to evaluate the efficacy of esophageal cancer patients with scales more objectively. In this paper, a lot of different MCID values were presented so that it can be easy and convenient to select by users.

\section{Abbreviations}

MCID: Minimal clinically important difference; QOL: Quality of life; PRO: Patient-reported outcomes; QLICPs: Quality of Life Instruments for Cancer Patients; ES: Esophageal cancer; QLICP-ES(V2.0): Quality of Life Instruments for Cancer Patients-Esophageal cancer(the second version); QLICP-GM(V2.0): Quality of Life Instruments for Cancer Patients-General Module(the second version); EORTC: European Organization for Research and Treatment of Cancer; QLQ-OES23: Quality of Life Questionnaire - Oesophageal 18; QLQ-C30: Quality of Life Questionnaire - Core 30; FACT-E: Functional Assessment of Cancer Therapy- Esophageal cancer questionnaire; ES: Effect-Size; SEM: Standard Error of the Measurement; RCI: Reliable Change Index; RS: Raw Score; SD: Standard-deviation; SDbaseline: Standard-deviation of the baseline score.

\section{Declarations}




\section{Acknowledgements}

In carrying out this research project, we have received substantial assistance from Prof. Gary Lyman from Hutchinson Institute for Cancer Outcomes Research, and Prof. David Cella, Benjamin J. Arnold and Hiramatsu Toshiko at the Center on Outcomes, Research, and Education (CORE), and many stalfs at the third affiliated hospital of Kunming Medical University (Yunnan Tumor Hospital), and Sun Yatsen University Cancer Center. We sincerely acknowledge all the support.

\section{Funding}

This study is supported by the National Natural Science Foundation of China $(71974040,81273185)$, the Features Innovative Projects of Key Platform and Major Scientific Research Project of Universities in Guangdong Province (2016KTSCX046, 2017KZDXM040). The funding bodies provided funds to support project development. The grant recipient (Chonghua Wan) designed the study, performed the data collection and data analyses, and extensively revised the manuscript.

\section{Availability of data and materials}

Not applicable.

\section{Authors' contributions}

CHW, GFL designed the study. TW, DDR, YBQ, JDZ and JYF performed the data collection. TW, DDR performed data analyses and drafted the manuscript. $\mathrm{CHW}$ revised the manuscript deeply. All authors contributed to interpreting the data, and have read and approved the final manuscript.

\section{Ethics approval and consent to participate}

The study protocol and the informed consent form were approved by the IRB (institutional review board) of the affiliated hospital of Guangdong Medical University (PJ2012052, YJYS2019010). The respondents were voluntary and provided written consent for participation.

\section{Consent for publication}

Not applicable.

\section{Competing interests}

The authors declare that they have no competing interests. 


\section{Author details}

${ }^{1}$ School of Humanities and Management, Research Center for Quality of Life and Applied Psychology, Guangdong Medical University, Dongguan 523808, CHINA.

${ }^{2}$ The Center for Response and Management of Emergence Public Health Event, the Center for Disease Control and Prevention of Yunnan Province, Kunming 650022, CHINA.

${ }^{3}$ Department of Thoracic Surgery, State Key Laboratory of Oncology in South China, Sun Yatsen University Cancer Center, Collaborative Innovation Center of Cancer Medicine, Guangzhou 510060, China

${ }^{4}$ The Third Affiliated Hospital of Kunming Medical University (Yunnan Tumor hospital), Kunming 650106, CHINA.

\section{References}

1. O'Farrell NJ, Feighery R, Picardo SL, Lynam-Lennon N, Biniecka M, McGarrigle SA, Phelan JJ, MacCarthy F, O'Toole D, Fox EJ, Ravi N, Reynolds JV, O'Sullivan J. Changes in mitochondrial stability during the progression of the Barrett's esophagus disease sequence. BMC Cancer. 2016;16:497.

2. Liu G, Zhao Y, Chen HL, Jia JR, Cheng XM, Wang FJ, Ji Q, Thorne R, Chen S, Liu XY. Analysis of Differentially Expressed Genes in a Chinese Cohort of Esophageal Squamous Cell Carcinoma. Journal of Cancer. 2020;11(13): 3783-3793.

3. Chen WQ, Li H, Sun KX, Zheng RS, Zhang SW, Zeng HM, Zou XN, Gu XY and He J. Report of Cancer Incidence and Mortality in China,2014. Chin J Oncol. 2018; 40(1): 5-13.

4. Li CX, Yang YY, Shen XC, Fan L, Liu KJ, Yang J, Chen DF and Lan FH. Analysis of endoscopic and clinical features of 81 cases of early esophageal cancer. Chin J Gastroenterol Hepatol. 2018;27(7):743746.

5. Barbieri A, Peyhardi J, Conroy T, Gourgou S, Lavergne C, Mollevi C. Item response models for the longitudinal analysis of health-related quality of life in cancer clinical trials. BMC Med Res Methodol. 2017;17(1):148.

6. Joseph KJ, Alvi R, Skarsgard D, Tonita J, Pervez N, Small C, Tai P. Analysis of health related quality of life (HRQoL) of patients with clinically localized prostate cancer, one year after treatment with external beam radiotherapy (EBRT) alone versus EBRT and high dose rate brachytherapy (HDRBT). Radiat Oncol. 2008;3:20.

7. Viklund P, Wengström Y, Rouvelas I, Lindblad M, Lagergren J. Quality of life and persisting symptoms after oesophageal cancer surgery. Eur J Cancer. 2006;42(10):1407-1414.

8. Blazeby JM, Alderson D, Winstone K, Steyn R, Hammerlid E, Arraras J and Farndon JR. Development of an EORTC questionnaire module to be used in quality of life assessment for patients with oesophageal cancer. The EORTC Quality of Life Study Group. Eur J Cancer. 1996;32A(11):1912-1917.

9. Yoo HJ, Kim SB, Yoon DH, Park SI, Kim JH, Cella D, Jung HY, Lee GH, Choi KD, Song HJ, Song HY, Shin $\mathrm{JH}, \mathrm{Cho}$ KJ. Translation and validation of Korean Functional Assessment of Cancer Therapy- 
Esophageal (FACT-E) scale with squamous cell carcinoma and chemoradiation-only patients. Qual Life Res.2012;21(8):1451-1457.

10. Qi YB, Li GF, Meng Q, Wan CH. Items selection in development of quality of life instrument for patients with esophageal cancer. Journal of International Oncology. 2010;37(7):554-556.

11. Qi YB, Li GF, Wan CH, Luo JH, Meng Q and Zhang XQ. Analysis of influence factors on quality of life in patients with esophageal cancer based on QLICP-ES. Chongqing Medicine. 2015;44(35):4995-4997.

12. Wan $\mathrm{CH}$, Yang Z, Meng Q, Feng CY, Wang HY, Tang XL. Development and Validation of the General Module of the System of Quality of Life Instruments for Cancer Patients (QLICP-GM). Int J Cancer.2008;122(1):190-196.

13. Yang Z, Luo JH, Meng Q, Li GF, Li XJ, Ding YL, Wan CH. Development and Validation of the System of Quality of Life Instruments for Cancer Patients: Head and Neck Cancer (QLICP-HN). Oral Oncology.2012; 48(8):737-746.

14. Guyatt GH, Walter S, Norman G. Measuring change over time: assessing the usefulness of evaluative instruments. J Chronic Dis.1987;40(2):171-178.

15. Jaeschke R, Singer J, Guyatt GH. Measurement of health status. Ascertaining the minimal clinically important difference. Control Clin Trials. 1989;10(4):407-415.

16. Zhang QQ, Qi YB, Wan CH, Li GF, Luo JH and Meng Q. The influence factors of quality of life in patients with esophageal cancer. Guangdong Medical Journal. 2013;34(12):1842-1844.

17. Wan $\mathrm{CH}, \mathrm{Yu} \mathrm{YL}$, Tan JF. Introductions on quality of life research measurements-assessmentsimprovements. Beijing: science press. 2016: 82-84.

18. Kazis LE, Anderson JJ, Meenan RF. Effect sizes for interpreting changes in health status. Med Care. 1989;27:S178-89.

19. Cohen J. Statistical power analysis for the behavioral sciences. 2nd ed,reprint. New York: Psychology Press; 2009.

20. Ekström M, Johnson MJ, Huang $C$ and Currow DC. Minimal clinically important differences in average, best, worst and current intensity and unpleasantness of chronic breathlessness. Eur Respir J.2020 in press.

21. Woaye-Hune P, Hardouin JB, Lehur PA, Meurette $G$ and Vanier A. Practical issues encountered while determining Minimal Clinically Important Difference in Patient-Reported Outcomes. Health Qual Life Outcomes. 2020;18(1):156.

22. Nunnally JC, Bernstein IH. Psychometric theory. 3rd ed. New York: McGraw-Hill; 1994.

23. Wyrwich KW, Tierney WM, Wolinsky FD. Further evidence supporting an SEM-based criterion for identifying meaningful intra-individual changes in health-related quality of life. J Clin Epidemiol. 1999;52:861-73.

24. Wyrwich KW, Nienaber NA, Tierney WM and Wolinsky FD. Linking clinical relevance and statistical significance in evaluating intra-individual changes in health-related quality of life. Med Care. 1999;37(5):469-478. 
25. Hu GQ, Huang QF, Huang ZN and Sun ZQ. Methods to determine minimal clinically important difference. Journal of central south university(medical science).2009;34(11):1058-1062.

26. Sloan DA, Donnelly MB, Schwartz RW, Felts JL, Blue AV and Strodel WE. The use of objective structured clinical examination (OSCE) for evaluation and instruction in graduate medical education. J Surg Res. 1996;63(1):225-230.

27. Mitchell CR, Vernon JA, Creedon TA. Measuring tinnitus parameters: loudness, pitch, and maskability. J Am Acad Audiol. 1993;4(3):139-151.

28. Gatchel RJ, Mayer TG, Choi Y, Chou R. Validation of a consensus-based minimal clinically important difference (MCID) threshold using an objective functional external anchor. Spine J. 2013;13(8):889-893.

29. Xu QA, Zhang CM, Wan $\mathrm{CH}$, Yang Z, Xu CZ and Chen Y. A computer simulation study on the calculation method of MCID. Chinese journal of health statistics. 2017;34(5):785-787.

30. Sun H, Xie Y and Li JS. Application of Minimal Clinically Important Difference in Quality of Life assessment tools for COPD patients. Chinese General Practice. 2015;18(23):2826-2829. 